\title{
ON THE VERY LONG TERM EVOLUTIONARY BEHAVIOR OF HYDROGEN-ACCRETING LOW MASS CO WHITE DWARFS
}

\author{
Luciano Piersanti ${ }^{1,2}$, Santi Cassisi ${ }^{2}$, Icko Iben, Jr. ${ }^{3}$, and Amedeo Tornambé ${ }^{2,4}$
}

Received —

\footnotetext{
${ }^{1}$ Dipartimento di Fisica, Università degli studi di Napoli "Federico II", Mostra d'Oltremare, Pad. 19, 80125 Napoli; lpiersanti@astrte.te.astro.it

${ }^{2}$ Osservatorio Astronomico di Teramo, Via M.Maggini 47, 64100 Teramo, Italy; cassisi@astrte.te.astro.it

${ }^{3}$ Astronomy and Physics Departments, University of Illinois, 1002 W. Green St., Urbana, IL 61801 icko@astro.uiuc.edu

${ }^{4}$ Dipartimento di Fisica, Università de L'Aquila, Via Vetoio, 67100 L'Aquila, Italy; tornambe@astrte.te.astro.it
} 


\begin{abstract}
Hydrogen-rich matter has been added to a carbon-oxygen white dwarf of initial mass $0.516 M_{\odot}$ at the rates $10^{-8}$ and $2 \times 10^{-8} M_{\odot} \mathrm{yr}^{-1}$, and results are compared with those for a white dwarf of the same initial mass which accretes pure helium at the same rates. For the chosen accretion rates, hydrogen burns in a series of recurrent mild flashes and the ashes of hydrogen burning build up a helium layer at the base of which a helium flash eventually occurs. In previous studies involving accretion at higher rates and including initially more massive white dwarfs, the diffusion of energy inward from the hydrogen shell-flashing region contributes to the increase in the temperature at the base of the helium layer, and the mass of the helium layer when the helium flash begins is significantly smaller than in a comparison model accreting pure helium; the helium shell flash is strong enough to cause the model to expand beyond its Roche lobe, but not strong enough to develop into a supernova explosion. In contrast, for the conditions adopted here, the temperature at the base of the helium layer becomes gradually independent of the deposition of energy by hydrogen shell flashes, and the mass of the helium layer when the helium flash occurs is a function only of the accretion rate, independent of the hydrogen content of the accreted matter. Several thousand hydrogen shell flashes must be followed before the helium flash takes place. Because of the high degeneracy at the base of the helium layer, temperatures in the flashing zone will rise without a corresponding increase in pressure, nuclear burning will continue until nuclear statistical equilibrium is achieved, and structural evolution will proceed hydrodynamically; the model will become a supernova, but not of the classical type Ia variety.
\end{abstract}


Subject headings: stars: evolution — stars: interiors — supernovae: general — stars: white dwarfs 


\section{Introduction}

Recently, Cassisi, Iben, and Tornambé (1998, hereinafter CIT) presented results of an extensive set of numerical computations describing the long term behavior of initially cool white dwarfs (WDs) accreting hydrogen-rich matter. Initial masses of $0.516 M_{\odot}$ and 0.8 $M_{\odot}$ were chosen and the accretion rate was varied over the range $10^{-8} M_{\odot} \mathrm{yr}^{-1}$ to $10^{-6}$ $M_{\odot} \mathrm{yr}^{-1}$. For intermediate accretion rates in this range, evolution was continued until a helium flash was initiated at the base of the layer of helium built up by hydrogen burning. One of the main results of the CIT experiments was a demonstration of how the thermal structure of the helium layer is modified by the injection of energy due to hydrogen burning compared with the thermal structure when pure helium is accreted at the same rate. In general, for the same initial mass, current mass, and accretion rate, the helium layer in the hydrogen-accreting model is hotter (see Fig. 8 in CIT) than in the helium-accreting model, and, when the helium flash begins, the total mass of the white dwarf accreting hydrogen is smaller (with a correspondingly lower density in the helium layer) than that of the white dwarf accreting pure helium. As a result, the helium flash in the hydrogen-accreting model is much less powerful than in the pure helium-accreting model 觇.

CIT also show that the envelopes of hydrogen-accreting white dwarfs of low mass which suffer a violent, albeit probably not dynamical, helium flash expand so much that, in the real world, most of the previously accreted matter may be lost from the system because of Roche-lobe overflow and common envelope activity.

In summary, in the CIT experiments, none of the white dwarfs accreting hydrogen-rich matter are able to reach the Chandrasekhar mass (and then explode their CO cores) or

\footnotetext{
${ }^{5}$ Note that, in the $3^{\mathrm{d}}$ line from the end of $\S 1$ in CIT, the words "helium" and "hydrogen" are mistakenly interchanged.
} 
to experience a "sub-Chandrasekhar" detonation in their helium layers. However, CIT conjecture that models of small mass which are accreting at rates which place them in the mild hydrogen-flash regime, but approaching the strong hydrogen-flash regime, may eventually experience a dynamical helium shell flash. Their conjecture is based on the fact that, for a fixed (small) initial white dwarf mass, the lower the accretion rate, the stronger is the final helium shell flash. By means of a simple extrapolation, CIT estimate a release of energy during the helium flash which could eventually turn the burning into a mildly dynamical event. The large number of hydrogen pulses required to reach the helium flash stage in models accreting at the relevant small rates persuaded CIT to postpone an explicit numerical investigation.

Over the past year, one relevant low- $\dot{M}$ calculation has been carried to the helium shell flash stage and a second calculation has reached the point where hydrogen-shell flashes are so strong as to seriously slow the rate of progress towards a helium-burning thermonuclear runaway. Even though not complete in the one case, both calculations establish that at the helium flash the mass of the helium layer and the violence of the event are much larger than expected on the basis of extrapolation from CIT results. The reason for this is that, in the present calculations, the helium layer becomes so massive prior to the helium flash that it acts as a buffer, insulating the base of the helium layer from the energy injected by hydrogen burning. Thus, the mass of the He layer at He ignition is independent of the composition of the accreted material.

\section{Results}

We have followed the very long term evolution of a white dwarf model of mass 0.516 $M_{\odot}$ accreting hydrogen at rates $10^{-8}$ and $2 \times 10^{-8} M_{\odot} \mathrm{yr}^{-1}$. The initial white dwarf has a central carbon-oxygen $(\mathrm{CO})$ core of mass $0.48 M_{\odot}$ and an outer helium layer of mass 0.036 
$M_{\odot}$. Details of the code, input physics, and numerical assumptions can be found in CIT and references therein. For both accretion rates, the accreting models undergo hydrogen shell flashes which require both fine zoning and very small time steps over a major portion of each pulse cycle. Typically, more than 2000 models are required to follow in detail one single pulse cycle during which the helium layer accretes about $10^{-4} M_{\odot}$ of fresh helium produced by hydrogen burning.

Some characteristics of the model accreting at the rate $2 \times 10^{-8} M_{\odot} \mathrm{yr}^{-1}$ are shown in Figure 1. There, the locations in mass of the hydrogen-helium discontinuity $\left(M_{\mathrm{H}-\text { shell }}\right)$, the point where the temperature is at a maximum in the helium layer $\left(M_{\mathrm{T}-\max }\right)$, and the maximum in the helium-burning energy-generation rate $\left(M_{\mathrm{He}-\text { shell }}\right)$ are shown as a function of the total mass (i.e., time). During the early part of the evolution, $M_{\mathrm{He}-\text { shell }}$ is relatively close to $M_{\mathrm{H}-\text { shell, }}$ as heat injected by hydrogen shell flashes diffuses inward, maintaining high temperatures. However, as temperatures and densities throughout the helium layer increase, the peak in the helium-burning energy-generation rate shifts to the base of the helium layer. This occurs quite suddenly at $M_{\text {tot }} \sim 0.556 M_{\odot}$.

Unfortunately, when the mass of the model reached $\sim 0.645 M_{\odot}$, in frustration at the real time consumed in following hydrogen shell flashes, we increased the accretion rate just enough that hydrogen began to burn in steady state. As may be deduced from Figure 10 in CIT, the required accretion rate is $\sim 6 \times 10^{-8} M_{\odot} \mathrm{yr}^{-1}$. When model mass reached $\sim 0.66$ $M_{\odot}$, the accretion rate was returned to $2 \times 10^{-8} M_{\odot} \mathrm{yr}^{-1}$ and hydrogen burning again proceeded by way of mild shell flashes. On comparing the shape of the $M_{\mathrm{T}-\max }$ curve before and after the episode of steady-state burning, it is clear that the model never completely recovered from the frustration-induced thermal pulse hiatus.

This failure to recover completely is demonstrated further in Figure 2 where the solid curve gives $\log T$ at the base of the helium layer as a function of $\log \rho$ there. The dashed 
curve in Figure 2 gives the same thing for the model which accretes pure helium at the rate $2 \times 10^{-8} M_{\odot} \mathrm{yr}^{-1}$. The two curves do not begin at the same point since quantities at the base of the helium layer were not stored during the early evolution of the model accreting hydrogen-rich matter. It is evident that, after the thermal pulse hiatus, the solid temperature-density curve proceeds at a higher temperature level than it did prior to the hiatus. Thus, the model retains a memory of the extra energy injected by increasing, even briefly, the accretion rate by a factor of 3. The dotted curve in Figure 2 is our conjecture for where the $\log T-\log \rho$ curve would be had we not introduced the thermal pulse hiatus.

In the model accreting hydrogen-rich matter, the helium-burning thermonuclear runaway occurs when model mass reaches $\sim 0.74 M_{\odot}$, corresponding to a helium layer of mass $\sim 0.26 M_{\odot}$. The helium accreting model experiences a thermonuclear runaway when model mass reaches $\sim 0.77 M_{\odot}$, corresponding to a helium layer of mass $\Delta M_{\mathrm{He}} \sim 0.29$ $M_{\odot}$. Our conjecture (represented by the dotted curve in Fig. 2) suggests that, had we not introduced the thermal pulse hiatus, the characteristics at the base of the helium layer in the hydrogen-accreting model would match those at the same point in the helium-accreting model prior to the onset of the thermonuclear runaway. Thus, the total model mass at the onset of the thermonuclear runaway is $\sim 0.77 M_{\odot}$ in both cases. The experiments with models accreting at the rate $10^{-8} M_{\odot} \mathrm{yr}^{-1}$ (see below) support our conjecture.

Extrapolating from the CIT results, we had expected that a helium-burning thermonuclear runaway would occur when $\Delta M_{\mathrm{He}} \sim 0.12 M_{\odot}$, less than half that given by the detailed computations. The reason for this difference is clear. The larger $\Delta M_{\mathrm{He}}$ is, the less sensitive (and lower) are temperatures in the deep interior of the helium layer to the details of hydrogen burning above the helium layer. The lower the accretion rate, the larger does $\Delta M_{\mathrm{He}}$ become before the helium flash begins. Thus, the lower the accretion rate, the closer does the pre-helium flash value of $\Delta M_{\mathrm{He}}$ for a hydrogen-accreting model approach 
the pre-helium flash value of $\Delta M_{\mathrm{He}}$ for its helium-accreting counterpart.

None of the models computed in the $\dot{M}=2 \times 10^{-8} M_{\odot} \mathrm{yr}^{-1}$ experiment enter the strong hydrogen-flash zone, therefore it has been possible to follow the early development of the helium flash up to the point that the outer edge of the convective layer formed initially near the base of the helium layer reaches the base of the hydrogen-rich envelope. Computations have been halted at this point, because a realistic calculation requires one to follow the time dependent diffusion of hydrogen into the helium convective zone until it reaches temperatures at which hydrogen can ignite. One might anticipate that hydrogen burning would force the development of a second, detached convective layer and that the accretor in a real counterpart would expand beyond its Roche lobe.

However, a more dramatic fate is in store for the model. At the start of the helium flash, the density $(\log \rho \sim 6.27)$ and temperature $(\log T>7.9)$ at the base of the helium layer are such that electrons are quite degenerate $\left(\epsilon_{\mathrm{F}} / k T>20\right)$, and one may expect that most of the nuclear energy liberated in the flashing zone will initially be converted into the thermal energy of ions and that, with temperatures exceeding $10^{9} \mathrm{~K}$, matter in the flashing zone will achieve nuclear statistical equilibrium before expansion becomes important. The net result will be a hydrodynamical event of supernova proportions (see the discussion and references in Iben \& Tutukov 1991).

Additional hints as to the final outcome can be derived by comparing the physical properties of our model with those of Woosley \& Weaver (1994; hereinafter WW) at runaway. The WW experiments do not include any with exactly the same initial mass and helium-accretion rate as we have used; however, we can take advantage of the analytical study of the physical properties of helium shell flashes provided by Fujimoto \& Sugimoto (1982; hereinafter FS) to find a relevant WW model. The parameters of our model in the $\Delta M_{\mathrm{He}, \mathrm{pk}} / M_{\odot}-M_{\mathrm{WD}}$ plane are slightly outside of the region for which the FS assumptions 
are strictly valid, but they are close enough that the FS results can be extrapolated with some confidence to estimate that the maximum energy-generation rate of our model is comparable to that of the WW "model 1": $M_{\mathrm{WD}, 0}=0.6 M_{\odot}, \dot{M}=2.5 \times 10^{-8} M_{\odot} \mathrm{yr}^{-1}$. This model produces an outgoing detonation in the helium layer and an inward moving sonic wave which eventually focuses at the center of the white dwarf causing an induced thermonuclear runaway. All in all, $\sim 0.9 \times 10^{51}$ ergs will be delivered in the event and the star will be completely disrupted.

The hydrogen accretion experiment with $\dot{M}=10^{-8} M_{\odot} \mathrm{yr}^{-1}$ has been followed, with no change in the accretion rate (i.e., no artificially introduced thermal pulse hiatus) until it has become clear that, long before the onset of the helium flash, the thermal structure near the base of the helium layer approaches that near the base of the helium layer in the companion model which accretes pure helium. The results which demonstrate this are shown in the temperature-density plane of Figure 3.

The calculation of the hydrogen-accreting model has been terminated because the hydrogen shell flashes have become so strong as to make following the evolution further impractical. At termination, $\Delta M_{\mathrm{He}} \sim 0.32 M_{\odot}$ and $M_{\text {tot }} \sim 0.80 M_{\odot}$.

Despite the termination, one can speculate reasonably confidently about further evolution. The hydrogen flashes are strong enough that the expansion of the hydrogen-rich envelope in a real analog extends well beyond the Roche lobe, with concomitant loss of matter of the sort experienced by classical novae. Thus, the real analog may never reach the helium-flashing stage. If it does reach the helium-flashing stage, one may use the properties of the helium-accreting model to predict that a supernova explosion terminates evolution. At the start of the helium flash, $\Delta M_{\mathrm{He}} \sim 0.50 M_{\odot}$ and $M_{\text {tot }} \sim 0.98 M_{\odot} ; \log \rho \sim 6.9$, $\log T \sim 7.8$, and $\epsilon_{\mathrm{F}} / k T>200$ at the base of the helium layer. These numbers imply that the supernova explosion terminating the life of the real analog, if it can reach this stage, 
will be even more violent than the one predicted for the model accreting hydrogen-rich matter at twice the rate.

In conclusion, we have shown that, for a restricted range of hydrogen-accretion rates and initial CO white dwarf masses, the outcome is a "sub-Chandrasekhar" supernova. In the absence of a hydrogen-rich companion, the real analog of the exploding white dwarf model would probably appear as a type I supernova (see, e.g., WW). That is, the hydrogen mass present at the top of the exploding dwarf $\left(\sim 10^{-4} M_{\odot}\right)$ is probably too small to produce detectable hydrogen lines in the spectrum. However, the nucleosynthetic products of a sub-Chandrasekhar explosion are such that the spectrum will depart considerably from that of a classical type Ia supernova (Höflich \& Khokhlov 1996, Nugent et al. 1997, several papers in Truran \& Niemeyer 1999). In the real analog, contamination of the supernova ejecta by hydrogen from the hydrogen-rich companion may even move the supernova out of the type I category entirely. The range of parameters for which an explosive outcome of the sort described here will occur suggests a variation in explosion energy of slightly less than a factor of 2. An estimate of what fraction of observed supernovae are of the sort discussed here requires a full fledged population synthesis study; such an estimate is beyond the scope of this paper.

\section{Influence of NCO Reactions on Flash Properties}

The referee has noted that, in the computations just described, the NCO reactions ${ }^{14} \mathrm{~N}\left(\mathrm{e}^{-}, \nu\right){ }^{14} \mathrm{C}(\alpha, \gamma){ }^{18} \mathrm{O}$ were not taken into account. There are several reasons for this omission. First, our starting white dwarf model is the end product of an evolutionary calculation with mass loss and, during the conversion of the helium core into a CO core,

nearly all of the ${ }^{14} \mathrm{~N}$ initially present in the core is destroyed via the ${ }^{14} \mathrm{~N}(\alpha, \gamma){ }^{18} \mathrm{O}(\alpha, \gamma){ }^{22} \mathrm{Ne}$ reactions, leaving only a very thin layer of unburned helium near the top. In fact, in 
the initial white dwarf model, there is no ${ }^{14} N$ at all below the point where the helium abundance is $\sim 0.7$, and, in our accretion models, helium ignition occurs much below this point. Secondly, a comparison between the results of Taam (1980) and of Woosley, Taam, \& Weaver (1986), who do not take the NCO reactions into account, and the results of WW, who do take them into account, shows that the inclusion of the NCO reactions lowers the critical mass of the accreted layer at the start of the triple- $\alpha$ runaway but does not prevent an explosive outcome, as the referee suggests might be the case. The latter three computations begin with a pure CO white dwarf which does not have the surface helium layer (in most of which there is no ${ }^{14} \mathrm{~N}$ ) which white dwarfs derived by evolutionary calculations possess. A comparison between results of the WW computations and those of Limongi \& Tornambè (1991), who used initial models derived by evolution, suggests that the influence of the NCO reactions on inducing the triple- $\alpha$ runaway may be quite small when evolutionary models are used, due to the lack of ${ }^{14} N$ nuclei over the inner part of the helium layer capping the CO core in the initial model.

Nevertheless, following the referee's suggestion, we have analyzed explicitly how inclusion of the NCO reactions alters our present results. We employ the Hashimoto et al. (1986) rate for the ${ }^{14} \mathrm{~N}\left(\mathrm{e}^{-}, \nu\right){ }^{14} \mathrm{C}$ reaction and the Caughlan, Fowler \& Zimmermann (1988) rate for the ${ }^{14} \mathrm{C}(\alpha, \gamma){ }^{18} \mathrm{O}$ reaction. The abundance of ${ }^{14} \mathrm{~N}$ in accreted material is taken as solar. As expected, NCO burning begins far from the base of the helium layer, inside the region where ${ }^{14} N$ nuclei are present at densities such that the threshold for electron capture on ${ }^{14} \mathrm{~N}$ is exceeded. As NCO burning becomes active, $3 \alpha$ reactions are already occurring at a non-negligible rate at the base of the helium layer. As accretion continues, the NCO-burning shell moves outwards in mass in consequence of the general increase in density in the helium shell and of the outward (in mass) motion of the base of the ${ }^{14} \mathrm{~N} \rightarrow{ }^{14} \mathrm{C}$ transition zone. 
While the peculiar behaviour of NCO burning is interesting in its own right (we will address this topic in some detail in a forthcoming paper), its actual effect on the outcome of our helium-accreting $\mathrm{CO}$ white dwarfs is rather small. As a matter of fact, in the $\dot{M}_{\mathrm{He}}=10^{-8} M_{\odot} \mathrm{yr}^{-1}$ experiment, the differences are negligible (less than a $2 \%$ change in both the mass of the accreted layer and in the ignition density when the thermonuclear runaway begins. Interestingly, the effect is slightly more important in the model accreting at the rate $\dot{M}_{H e}=2 \times 10^{-8} M_{\odot} \mathrm{yr}^{-1}$, but, even so, the differences in ignition density and in the critical mass of the helium layer at the $3 \alpha$ runaway are less than $10 \%$. By interpolation, we estimate that, for an accretion rate of $\dot{M}_{H e}=1.58 \times 10^{-8} M_{\odot} \mathrm{yr}^{-1}$, triple- $\alpha$ runaway conditions will be essentially identical to those found in the $\dot{M}_{H e}=2 \times 10^{-8} M_{\odot} \mathrm{yr}^{-1}$ model without the NCO reactions. Our previous arguments for a dynamic event are thus still valid.

It is easy to understand why, the larger the accretion rate, the more effective are the NCO reactions in reducing the critical mass of the helium layer when the $3 \alpha$ runaway begins, relative to the case when the NCO reactions are neglected. With or without the NCO reactions, increasing the accretion rate leads to more rapid heating and a decrease in the critical mass for helium ignition. Thus, with the NCO reactions included, the larger the accretion rate, the closer to the base of the helium layer does the NCO-burning shell form. The closer the NCO-burning shell is to the base of the helium layer, the more rapidly does heat from this shell diffuse to the base of the helium layer and the sooner are conditions reached for the triple- $\alpha$ runaway to begin. A similar phenomenon occurs in models accreting hydrogen-rich matter at a high rate (CIT); the larger the accretion rate, the greater is the influence of heat diffusing from the hydrogen shell flashing region in inducing helium-burning flashes.

The most important result of the experiments described in this section is that explosive 
outcomes are produced in the range of $\dot{M}$ examined, even when NCO reactions are taken into account, thus confirming previous results by WW. 


\section{REFERENCES}

Cassisi, S., Iben, I. Jr., \& Tornambé, A., 1998, ApJ, 496, 376

Caughlan, G.R., Fowler, W.A., \& Zimmerman, B., 1988, Atomic Data \& Nuclear Data Tables, vol. 40, n.20, p. 284

Fujimoto, M. Y., \& Sugimoto, D., 1982, ApJ, 257, 291

Hashimoto, M. A., Nomoto, K.I., Arai, K., \& Kaminisi, K., 1986, ApJ, 307, 687

Höflich, P. \& Khokhlov, A., 1996, ApJ, 457, 500

Iben, I. Jr., \& Tutukov. A. V., 1991, ApJ, 370, 615

Nomoto, K., 1982, ApJ, 257, 780

Limongi, M., \& Tornambé, A. 1991, ApJ, 371, 317

Nugent, P., Baron, E., Branch, D., Fisher, A., \& Hauschildt, P. H., 1997, ApJ, 485, 812

Taam, R.E., 1980, ApJ, 237, 142

Truran, J. W., \& Niemeyer, J. 1999, eds., Type Ia Supernovae, Theory and Cosmology, in press

Woosley, S.E., Taam, R.E. \& Weaver, T.A., 1986, ApJ, 301, 601

Woosley, S.E., \& Weaver, T.A., 1994, ApJ, 423, 371 
Fig. 1. - Several characteristics of a model white dwarf accreting hydrogen-rich material at the rate $\dot{M}=2 \times 10^{-8} M_{\odot} \mathrm{yr}^{-1}$ are shown as a function of the total mass (i.e., of the time). Characteristics are the locations in mass of the hydrogen-helium discontinuity $\left(M_{\mathrm{H}-\text { shell }}\right)$, the point where the temperature is at a maximum in the helium layer $\left(M_{\mathrm{T}-\max }\right)$, and the maximum in the helium-burning energy-generation rate $\left(M_{\mathrm{He}-\text { shell }}\right)$.

Fig. 2.- The evolution in temperature $T$ and density $\rho$ at the base of the helium layer in model white dwarfs accreting hydrogen-rich matter (solid line) and pure helium (dashed line) at the rate $\dot{M}=2 \times 10^{-8} M_{\odot} \mathrm{yr}^{-1}$. The dotted curve is an estimate of behavior had the hydrogen flashes not been temporarily suppressed by increasing the accretion rate by a factor of three as total mass is increased from $\sim 0.645 M_{\odot}$ to $\sim 0.66 M_{\odot}$.

Fig. 3.- Same as Fig. 1 when $\dot{M}=2 \times 10^{-8} M_{\odot} \mathrm{yr}^{-1}$, except that the accretion rate has been kept strictly constant for the entire evolution of the hydrogen-accreting model. 


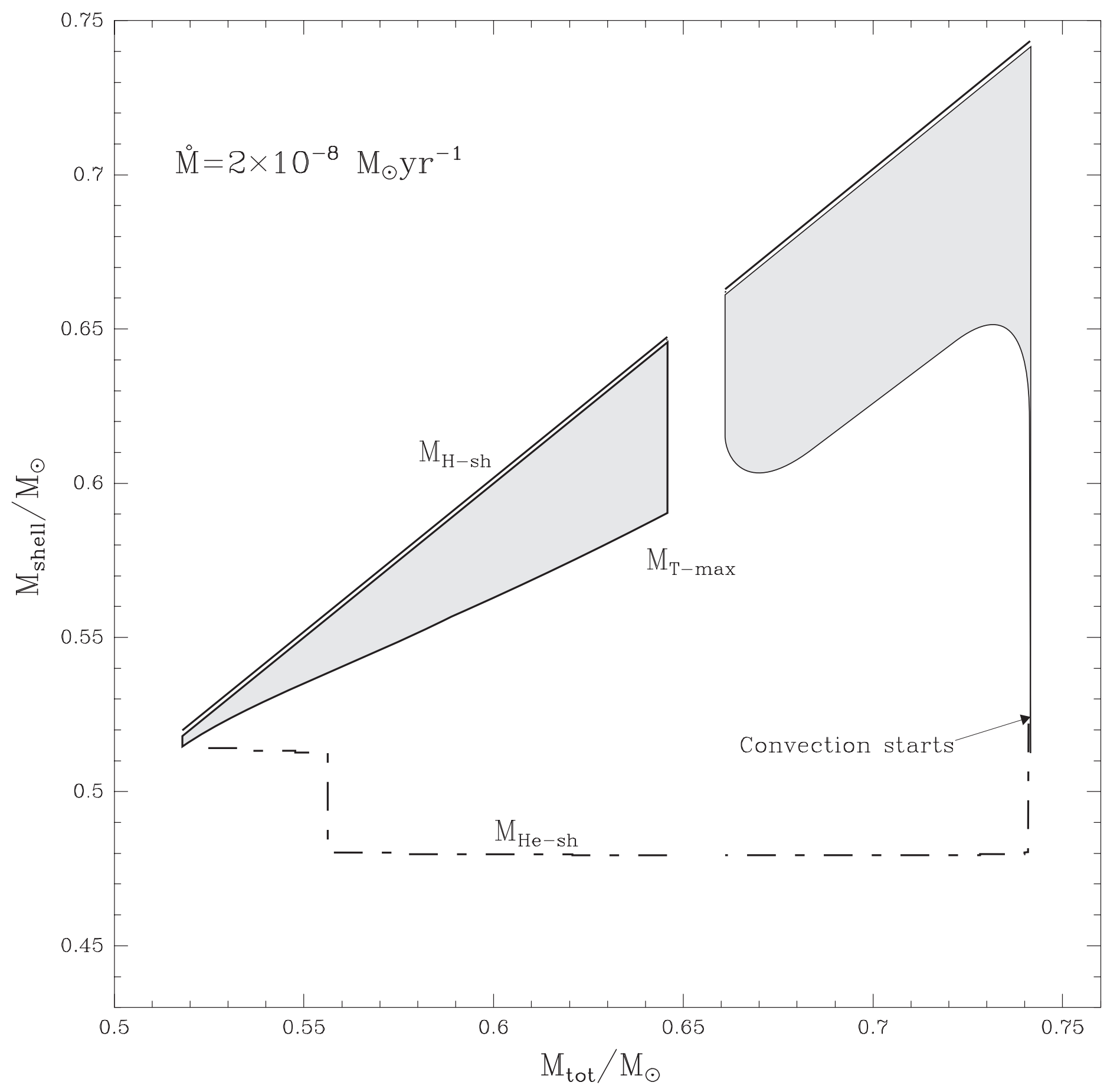




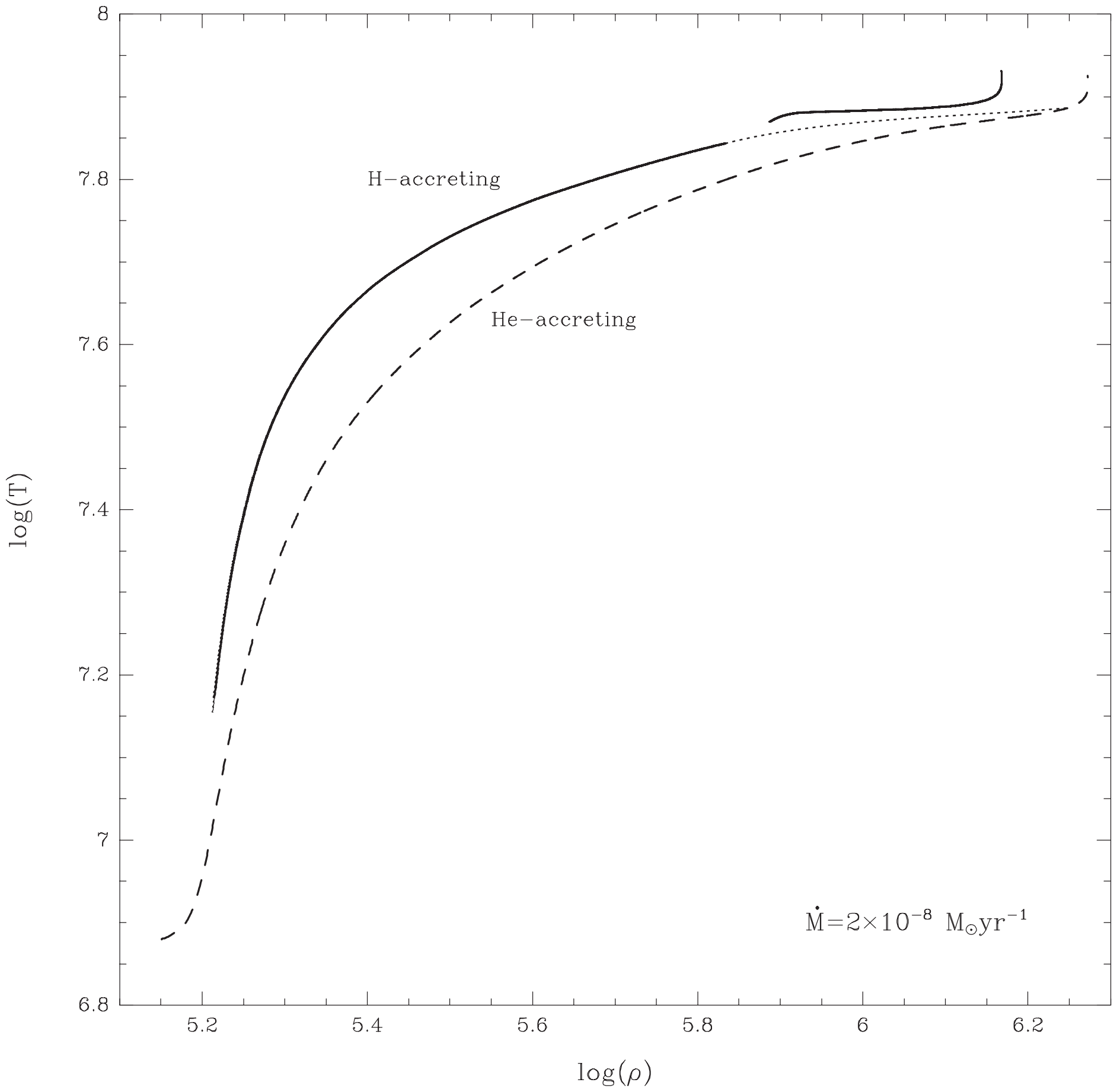




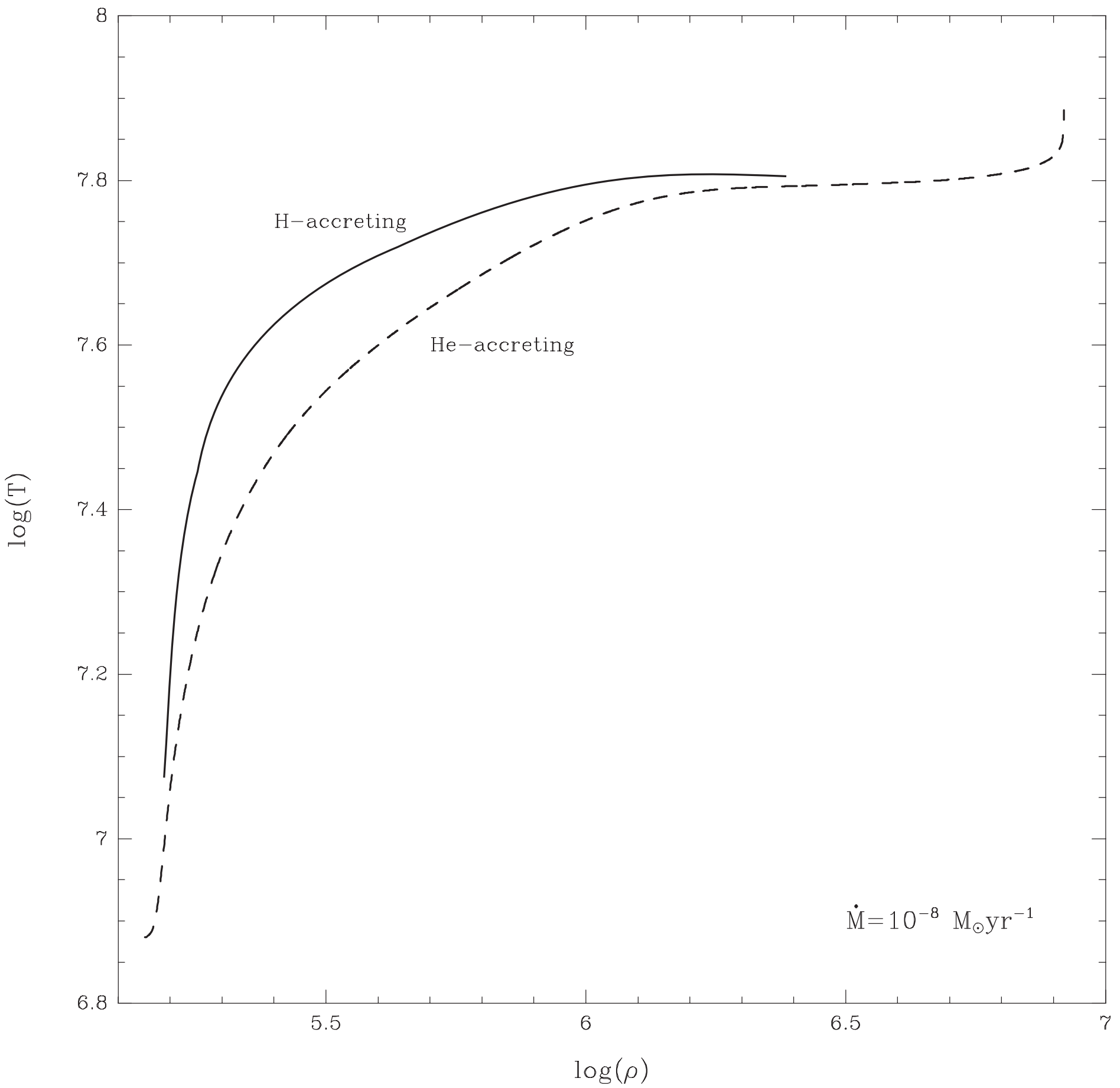

